\title{
OPTIMALITY AND DUALITY WITHOUT A CONSTRAINT QUALIFICATION FOR MINIMAX PROGRAMMING
}

\author{
Houchun Zhou and WenYu Sun
}

\begin{abstract}
Without the need of a constraint qualification, we establish the optimality necessary and sufficient conditions for generalised minimax programming. Using these optimality conditions, we construct a parametric dual model and a parameter-free mixed dual model. Several duality theorems are established.
\end{abstract}

\section{INTRODUCTION}

Consider the generalised minimax programming:

$$
(P) \quad \min _{x \in S} \sup _{y \in Y} \phi(x, y)
$$

where $S$ is nonempty subset of $R^{n}$ defined by

$$
S=\left\{x \in R^{n}: h_{j}(x) \leqslant 0, j=1,2, \ldots, m\right\} .
$$

$Y$ is a compact subset of $R^{n}, \phi(x, y): R^{n} \times Y \rightarrow R$ is a convex function with respect to $x$, and $h_{j}(x): R^{n} \rightarrow R, j=1, \ldots, m$ are convex functions, for each $x \in R^{n}$, and $\phi(x, y)$ is upper semi-continuous with respect to $y$.

Ben-Israel, Ben-Tal, and Zlobec presented some necessary and sufficient optimality conditions for (scalar) convex programming problems without any constraint qualification in [2], and Egudo, Weir, and Mond gave some necessary and sufficient optimality conditions for multi-objective convex programming problems without any constraint qualification in [4]. Latter, Mond[7], Ben-Tal, and Zlobec [2], Egudo [4], Weir [8, 9] used these optimality conditions in designing various dual models and established several duality theorems without any constraint qualification for the (convex or generalised convex, single-objective or multi-objective) mathematical programming.

Motivated by [3, 2], Lai, Liu and Tanaka [6] established some optimality necessary and sufficient conditions for the generalised fractional programming without any constraint qualification and constructed a parametric dual model and two parameter-free dual models.

Received 15th July, 2002

This work was supported by National Natural Science Foundation of China.

Copyright Clearance Centre, Inc. Serial-fee code: 0004-9727/03 \$A2.00+0.00. 
In this paper, we establish the optimality necessary and sufficient conditions for generalised minimax programming (P) without the need of a constraint qualification. Using these optimality conditions and the ideas of Bector [1], we construct a parametric dual model and a parameter-free mixed dual model, whereas the latter model unifies the two parameter-free dual models of Lai, Liu andk Tanaka in [6]. Several duality theorems are established, subsequently, one of the problems posed by Lai, Liu and Tanaka [6] is solved.

\section{NOTATIONS AND PRELIMINARY RESULTS}

In this section, we first introduce some notation. Let $J=\{1,2, \ldots, m\}$, and define

$$
J(x)=\left\{j \in J \mid h_{j}(x)=0\right\}
$$

as the set of active indices at $x$. The minimal index set of the binding constraints at $x$ for $S$ is

$$
J=\left\{j \in J \mid h_{j}(x)=0, \text { for all } x \in S\right\}
$$

We also denote

$$
J^{<}(x)=J(x) \backslash J^{=}=\left\{j \in J(x) \mid \exists x_{i} \in S \text { to satisfy } h_{j}\left(x_{i}\right)<0\right\} .
$$

Define the set

$$
S^{=}=\left\{x \in R^{n} \mid h_{j}(x) \leqslant 0, j \in J^{=}\right\}
$$

with the convention

$$
S^{=}=R^{n}, \text { if } J^{=}=\emptyset .
$$

Since $J^{=} \subset J$, the set $S^{=} \supset S$. Obviously,

$$
S^{=}=\left\{x \in R^{n} \mid h_{j}(x)=0, j \in J^{=}\right\}
$$

Definition 2.1. ([6]) For a function $h: R^{n} \mapsto R$ and a point $x \in \operatorname{dom}(h)$, the cone of directions of constancy at $x$ with respect to the function $h$ is defined by

$$
\begin{aligned}
& D_{h}^{=}(x)=\left\{d \in R^{n} \mid \text { there exists an } \bar{\alpha}\right. \text { such that } \\
& \qquad h(x+\alpha d)=h(x) \text {, for every } 0<\alpha<\bar{\alpha}\} .
\end{aligned}
$$

We use the following notation:

$$
D_{j}^{=}(x)=D_{h_{j}}^{=}(x), D_{J}^{=}(x)=\bigcap_{j \in J} D_{j}^{=}(x) .
$$

If $J=\emptyset$, we define $D_{\emptyset}^{=}(x)=R^{n}$. 
Definition 2.2. ([6]) Let $M \subset R^{n}$ be a nonempty subset. The positive dual cone $M^{*}$ of $M$ is defined by

$$
M^{*}=\left\{d \in R^{n} \mid d^{T} x \geqslant 0, \text { for all } x \in M\right\} .
$$

LEMMA 2.3. ([7]) If $x \in S$ and $u \in S^{=}$, then

$$
(x-u)^{T} d \geqslant 0, \text { for all } d \in\left[D_{J}^{=}=(x)\right]^{*} .
$$

Let $A: R^{n} \mapsto R$ be a convex continuous function. We consider the following scalar minimisation problem:

$$
\text { (SP) } \min \{A(x) \mid x \in S\} \text {. }
$$

Lemma 2.4. ([2, Corollary 3.7]) Let $x_{0} \in S$. Then, $x_{0}$ is an optimal solution of (SP) if, and only if, there exist $\gamma_{j} \geqslant 0, j \in J^{<}\left(x_{0}\right)$, such that

$$
0 \in \partial A\left(x_{0}\right)+\sum_{j \in J<\left(x_{0}\right)} \gamma_{j} \partial h_{j}\left(x_{0}\right)-\left[D_{J}^{=}=(x)\right]^{*} .
$$

Let $Y$ is a infinite compact set, $f_{y}, y \in Y$ are convex functions, we assume that

$$
\begin{gathered}
f(x):=\sup \left\{f_{y}(x) \mid y \in Y\right\}<+\infty \quad \text { for all } x \in R^{n}, \\
Y(x)=\left\{y \in Y \mid f_{y}(x)=f(x)\right\} .
\end{gathered}
$$

Lemma 2.5. ([5, Theorem 4.4.2]) Assume that $f_{y}, y \in Y$ are convex functions, $Y$ is a infinite compact set, on which the functions $y \mapsto f_{y}(x)$ are upper semi-continuous for each $x \in R^{n}$. Then

$$
\partial f(x)=\operatorname{co}\left\{\cup \partial f_{y}(x), y \in Y(x)\right\} .
$$

In the following, we shall consider elements of the set $R_{+}^{(Y)}$ defined as

$$
R_{+}^{(Y)}:=\left\{\lambda: Y \rightarrow R_{+} \mid \lambda_{y}=0, \quad \text { for all } y \text { except for a finite number }\right\} .
$$

Lemma 2.6. For each $x \in S$, one has

$$
f(x)=\sup _{y \in Y}[\phi(x, y)]=\sup _{\beta \in U}\left[\sum_{y \in D(\beta)} \beta_{y} \phi(x, y)\right],
$$

where $U=\left\{\beta \in R_{+}^{(Y)} \mid \sum_{y \in D(\beta)} \beta_{y}=1\right\}, D(\beta)=\left\{y \in Y \mid \beta_{y} \neq 0\right\}$.

Proof: For arbitrary $y_{0} \in Y$, let $\bar{\beta}=\left(\bar{\beta}_{y}\right)$, where $\bar{\beta}_{y}=1$, if $y=y_{0}$; otherwise, $\bar{\beta}_{y}=0$, thus $\bar{\beta} \in U$ and

$$
\phi\left(x, y_{0}\right)=\sum_{y \in D(\bar{\beta})} \bar{\beta}_{y} \phi(x, y) \leqslant \sup _{\beta \in U}\left[\sum_{y \in D(\beta)} \beta_{y} \phi(x, y)\right] .
$$


By the arbitrariness of $y_{0} \in Y$, we derive

$$
\sup _{y \in Y} \phi(x, y) \leqslant \sup _{\beta \in U}\left[\sum_{y \in D(\beta)} \beta_{y} \phi(x, y)\right] \text {. }
$$

On the other hand, For arbitrary $\bar{\beta}=\left(\bar{\beta}_{y}\right) \in U$, we have

$$
\sum_{y \in D(\bar{\beta})} \bar{\beta}_{y} \phi(x, y) \leqslant \max \{\phi(x, y) \mid y \in D(\bar{\beta})\} \leqslant \sup _{y \in Y} \phi(x, y) .
$$

By the arbitrariness of $\bar{\beta} \in U$, it holds that

$$
\sup _{\beta \in U}\left[\sum_{y \in D(\beta)} \beta_{y} \phi(x, y)\right] \leqslant \sup _{y \in Y}(\phi(x, y))
$$

Thus

$$
\sup _{y \in Y} \phi(x, y)=\sup _{\beta \in U}\left[\sum_{y \in D(\beta)} \beta_{y} \phi(x, y)\right]
$$

\section{Optimality NECESSARY AND SUfFicient CONDITION FOR (P)}

In this section, we shall establish optimality necessary and sufficient conditions for the generalised minmax programming problem $(\mathrm{P})$.

In the following, we suppose that for each $v \in R_{+}$,

$$
f(x)=\sup \{\phi(x, y) \mid y \in Y\}<+\infty \text { for all } x \in R^{n}
$$

and denote:

$$
\begin{aligned}
L\left(x_{0}\right) & =\left\{y \in Y \mid \phi\left(x_{0}, y\right)=\sup _{y \in Y} \phi\left(x_{0}, y\right)\right\} \\
R_{+}^{\left(L\left(x_{0}\right)\right)} & =\left\{\alpha: L\left(x_{0}\right) \rightarrow R_{+} \mid \alpha_{y}=0, \text { for all } y \text { except for a finite number }\right\}
\end{aligned}
$$

and $U_{0}=\left\{\alpha \in R_{+}^{\left(L\left(x_{0}\right)\right.} \mid \sum_{y \in D_{0}(\alpha)} \alpha_{y}=1\right\}, \quad D_{0}(\alpha)=\left\{y \in L\left(x_{0}\right) \mid \alpha_{y} \neq 0\right\}$.

Based on Lemmas 2.4 and 2.5, we can get the following result.

THEOREM 3.1. $x_{0} \in S$ is an optimal solution of $(P)$ if, and only if, there exist $\alpha \in U_{0}$, and $\gamma_{j} \geqslant 0, j \in J^{<}\left(x_{0}\right)$, such that

$$
0 \in \sum_{y \in D_{0}(\alpha)} \alpha_{y}\left[\partial \phi(\cdot, y)\left(x_{0}\right)\right]+\sum_{j \in J<\left(x_{0}\right)} \gamma_{j} \partial h_{j}\left(x_{0}\right)-\left[D_{j=}^{=}\left(x_{0}\right)\right]^{*}
$$

PROOF: If $x_{0}$ is an optimal solution of (P), then, by lemma 2.4 , there exist $\gamma_{j}$ $\geqslant 0, j \in J^{<}\left(x_{0}\right)$, such that

$$
0 \in \partial \sup _{y \in Y}[\phi(\cdot, y)]\left(x_{0}\right)+\sum_{j \in J^{<}\left(x_{0}\right)} \gamma_{j} \partial h_{j}\left(x_{0}\right)-\left[D_{J}^{=}=\left(x_{0}\right)\right]^{*} .
$$


It follows from Lemma 2.5 that there exists $\alpha \in U_{0}$, such that

$$
0 \in \sum_{y \in D_{0}(\alpha)} \alpha_{y}[\partial \phi(\cdot, y)]\left(x_{0}\right)+\sum_{j \in J<\left(x_{0}\right)} \gamma_{j} \partial h_{j}\left(x_{0}\right)-\left[D_{J=}^{=}\left(x_{0}\right)\right]^{*}
$$

Conversely, if there exist $\alpha \in U_{0}$, and $\gamma_{j} \geqslant 0, j \in J^{<}\left(x_{0}\right)$, such that

$$
0 \in \sum_{y \in D_{0}(\alpha)} \alpha_{y}[\partial \phi(\cdot, y)]\left(x_{0}\right)+\sum_{j \in J<\left(x_{0}\right)} \gamma_{j} \partial h_{j}\left(x_{0}\right)-\left[D_{J=}^{=}\left(x_{0}\right)\right]^{*},
$$

then from Lemma 2.5, it follows that

$$
\sum_{y \in D_{0}(\alpha)} \alpha_{y}[\partial \phi(\cdot, y)]\left(x_{0}\right) \subset \operatorname{co}\left\{\bigcup_{y \in L\left(x_{0}\right)} \partial[\phi(\cdot, y)]\left(x_{0}\right)\right\}=\partial \sup _{y \in Y}[\phi(\cdot, y)]\left(x_{0}\right)
$$

So we obtain

$$
0 \in \partial \sup _{y \in Y}[\phi(\cdot, y)]\left(x_{0}\right)+\sum_{j \in J<\left(x_{0}\right)} \gamma_{j} \partial h_{j}\left(x_{0}\right)-\left[D_{J}^{=}=\left(x_{0}\right)\right]^{*}
$$

By lemma 2.4 we can deduce that $x_{0}$ is an optimal solution of $(\mathrm{P})$.

For $\alpha \in U$, we denote by

$$
\begin{gathered}
\alpha^{T} \Phi(x, y)=\sum_{y \in D(\alpha)} \alpha_{y} \phi(x, y), \quad \gamma^{T} H(x)=\sum_{j=1}^{m} \gamma_{j} h_{j}(x) \\
\partial\left[\alpha^{T} \Phi(\cdot, y)\right](x)=\sum_{y \in D(\alpha)} \alpha_{y}[\partial \phi(\cdot, y)](x), \quad \partial\left[\gamma^{T} H\right](x)=\sum_{j=1}^{m} \gamma_{j} \partial h_{j}(x) .
\end{gathered}
$$

Corollary 3.2. (Optimality Necessary Condition) If $x_{0} \in S$ is an optimal solution of $(P)$, then there exist $\alpha \in U$, and $\gamma \in R_{+}^{m}$, such that

$$
\begin{gathered}
0 \in \partial\left[\alpha^{T} \Phi(\cdot, y)\right]\left(x_{0}\right)+\partial\left[\gamma^{T} H\right]\left(x_{0}\right)-\left[D_{J}^{=}=\left(x_{0}\right)\right]^{*}, \\
\alpha^{T} \phi\left(x_{0}, y\right)=f\left(x_{0}\right), \\
\gamma^{T} H\left(x_{0}\right)=0 .
\end{gathered}
$$

Proof: In the proof of Theorem 3.1, if we set

$$
\alpha_{y}=0, y \in Y \backslash L\left(x_{0}\right) \text {, and } \gamma_{j}=0, j \in J \backslash J^{<}\left(x_{0}\right) \text {, }
$$

then (3.1)-(3.3) hold.

Theorem 3.3. (Optimality Sufficient Condition) Let $x_{0} \in S$ and $f\left(x_{0}\right)$ $=\sup _{y \in Y}\left[\phi\left(x_{0}, y\right)\right]$. Assume that there exist $\alpha \in U$ and $\gamma \in R_{+}^{m}$ such that the expressions (3.1)-(3.3) hold. Then, $x_{0}$ is an optimal solution of $(P)$. 
Proof: Suppose to the contrary that $x_{0}$ is not an optimal solution of (P). Then there exists a feasible solution $x_{1} \in S$ such that

$$
\sup _{y \in Y}\left[\phi\left(x_{1}, y\right)\right]<f\left(x_{0}\right) .
$$

From Lemma 2.6, we get

$$
\alpha^{T} \Phi\left(x_{1}, y\right) \leqslant \sup _{\beta \in U}\left[\sum_{y \in D(\beta)} \beta_{y} \phi(x, y)\right]<f\left(x_{0}\right) .
$$

Combining with relation (3.2) yields

$$
\alpha^{T} \Phi\left(x_{1}, y\right)<\alpha^{T} \Phi\left(x_{0}, y\right)
$$

Since $x_{1} \in S, \gamma \in R_{+}^{m}$, using (3.3), we have

$$
\gamma^{T} H\left(x_{1}\right) \leqslant 0=\gamma^{T} H\left(x_{0}\right)
$$

Hence,

$$
\alpha^{T} \Phi\left(x_{1}, y\right)+\gamma^{T} H\left(x_{1}\right)<\alpha^{T} \Phi\left(x_{0}, y\right)+\gamma^{T} H\left(x_{0}\right)
$$

By (3.1), there exist

$$
\xi \in \partial\left[\alpha^{T} \Phi(\cdot, y)\right]\left(x_{0}\right), \sigma \in \partial\left[\gamma^{T} H\right]\left(x_{0}\right), \tau \in\left[D_{J=}^{=}=\left(x_{0}\right)\right]^{*},
$$

such that

$$
\xi+\sigma-\tau=0 .
$$

By the convexity of functions $\alpha^{T} \Phi(\cdot, y), \gamma^{T} H$, we have

$$
\alpha^{T} \Phi\left(x_{1}, y\right)-\alpha^{T} \Phi\left(x_{0}, y\right)+\gamma^{T} H\left(x_{1}\right)-\gamma^{T} H\left(x_{0}\right) \geqslant\left(x_{1}-x_{0}\right)^{T}(\xi+\sigma)=\left(x_{1}-x_{0}\right)^{T} \tau \geqslant 0 .
$$

This contradicts inequality (3.4). So, $x_{0}$ is an optimal solution of (P).

\section{Parametric dual Model}

In this section, we consider the following parametric dual problem:

(D1) $\quad \max \lambda$

$$
\begin{array}{ll}
\text { subject to } & 0 \in \partial\left[\alpha^{T} \Phi(\cdot, y)\right](u)+\partial\left[\gamma^{T} H\right](u)-\left[D_{J}^{=}(u)\right]^{*}, \\
& \alpha^{T} \Phi(u, y)+\gamma^{T} H(u) \geqslant \lambda \\
& \alpha \in U, \gamma \in R_{+}^{m} \\
& u \in S^{=}
\end{array}
$$

We denote $S_{1}$ the set of all feasible solutions $(u, \alpha, \gamma, \lambda) \in S^{=} \times U \times R_{+}^{m} \times R_{+}$of Problem (D1). Then, a weak duality theorem is established as follows. 
Theorem 4.1. (Weak Duality) Let $x \in S$ and $(u, \alpha, \gamma, \lambda) \in S_{1}$. Then

$$
\lambda \leqslant \sup _{y \in Y} \phi(x, y) .
$$

PROOF: By relation (4.1), there exist

$$
\xi \in \partial\left[\alpha^{T} \Phi(\cdot, y)\right](u), \quad \sigma \in \partial\left[\gamma^{T} H\right](u), \quad \tau \in\left[D_{J=}^{=}(u)\right]^{*},
$$

such that

$$
\xi+\sigma-\tau=0 .
$$

By the convexity of functions $\alpha^{T} \Phi(\cdot, y), \gamma^{T} H$, we have

$$
\alpha^{T} \Phi(x, y)-\alpha^{T} \Phi(u, y)+\gamma^{T} H(x)-\gamma^{T} H(u) \geqslant(x-u)^{T}(\xi+\sigma)=(x-u)^{T} \tau \geqslant 0 .
$$

It follows that

$$
\alpha^{T} \Phi(x, y)+\gamma^{T} H(x) \geqslant \alpha^{T} \Phi(u, y)+\gamma^{T} H(u) .
$$

From (4.2), (4.5) and $\gamma^{T} H(x) \leqslant 0$, we get

$$
\alpha^{T} \Phi(x, y) \geqslant \alpha^{T} \Phi(u, y)+\gamma^{T} H(u) \geqslant \lambda .
$$

Hence, from Lemma 2.6, one has

$$
\sup _{y \in Y} \phi(x, y)=\sup _{\beta \in U}\left[\sum_{y \in D(\beta)} \beta_{y} \phi(x, y)\right] \geqslant \alpha^{T} \Phi(x, y) \geqslant \lambda .
$$

TheOREM 4.2. (Strong Duality) Let $\bar{u} \in S$ be an optimal solution of $(P)$. Then there exist $\bar{\alpha} \in U, \bar{\gamma} \in R_{+}^{m}, \bar{\lambda} \in R_{+}$such that $(\bar{u}, \bar{\alpha}, \bar{\gamma}, \bar{\lambda}) \in S_{1}$ is an optimal solution of $(D 1)$, and the optimal values of $(P)$ and (D1) are equal.

Proof: By Corollary 3.2, there exist $\bar{\alpha} \in U, \bar{\gamma} \in R_{+}^{m}, \bar{\lambda} \in R_{+}$such that $(\bar{u}, \bar{\alpha}, \bar{\gamma}, \bar{\lambda})$ is a feasible solution for (D1) and $\bar{\lambda}=f(\bar{u})$, by Theorem 4.1 , we derive that $(\bar{u}, \bar{\alpha}, \bar{\gamma}, \bar{\lambda})$ is an optimal solution for (D1), and the optimal values of (P) and (D1) are equal.

Theorem 4.3. (Strict Converse Duality) Let $\bar{x} \in S$ and $(\bar{u}, \bar{\alpha}, \bar{\gamma}, \bar{\lambda}) \in S_{1}$ be optimal solution of $(P)$ and (D1), respectively. If $\bar{\alpha}^{T} \Phi(\cdot, y), \bar{\gamma}^{T} H(\cdot)$ are convex and one of them is strictly convex at $\bar{u}$, then $\bar{x}=\bar{u}$; that is, $\bar{u}$ is an optimal solution of $(P)$ and $f(\bar{x})=\bar{\lambda}$.

Proof: Suppose to the contrary that $\bar{x} \neq \bar{u}$. From Theorem 3.2, we know that there exist $\bar{\alpha}_{1} \in U, \bar{\gamma}_{1} \in R_{+}^{m}, \bar{\lambda}_{1} \in R_{+}$such that $\left(\bar{x}, \bar{\alpha}_{1}, \bar{\gamma}_{1}, \bar{\lambda}_{1}\right) \in S_{1}$ is an optimal solution of (D1) with the optimal value $\bar{\lambda}_{1}=f(\bar{x})$. Similar to the proof of Theorem 4.1, we can obtain the strict inequality $f(\bar{x})>\bar{\lambda}$ which contradicts that $f(\bar{x})=\bar{\lambda}_{1}=\bar{\lambda}$. The proof is complete. 


\section{MiXed-TyPE DUAL MODEL}

In this section, we shall introduce the parameter-free mixed type duality for (P) and establish several mixed duality theorems. The following dual problem is called a Mixed-type dual problem:

$$
\begin{array}{cl}
(M D) & \sup \alpha^{T} \Phi(u, y)+\gamma_{J_{1}}^{T} H_{J_{1}}(u) \\
\text { subject to } & 0 \in \partial\left[\alpha^{T} \Phi(\cdot, y)\right](u)+\partial\left[\gamma^{T} H\right](u)-\left[D_{J}^{=}=(u)\right]^{*}, \\
& \gamma_{j} h_{j}(u) \geqslant 0, j \in J_{2}, \\
& \alpha \in U, \gamma_{j} \geqslant 0, j \in J, u \in S^{=}
\end{array}
$$

where $J_{1}$ is a subset of $J=\{1, \ldots, m\}, J_{2}=J \backslash J_{1}$.

We denote the set of all feasible solution $(u, \alpha, \gamma) \in S^{=} \times U \times R_{+}^{m}$ of problem (MD) by $S_{2}$. In the following, we shall prove the weak duality, strong duality, and strict converse duality theorems.

ThEOREM 5.1. (Weak Duality) Let $x \in S$ and $(u, \alpha, \gamma) \in S_{2}$. Then

$$
f(x) \geqslant \alpha^{T} \Phi(u, y)+\gamma_{J_{1}}^{T} H_{J_{1}}(u)
$$

Proof: By (5.1), there exist

$$
\begin{array}{ll}
q \in \partial\left[\alpha^{T} \Phi(\cdot, y)\right](u), & r \in \partial\left[\gamma_{J_{1}}^{T} H_{J_{1}}\right](u), \\
e \in \partial\left[\gamma_{J_{2}}^{T} H_{J_{2}}\right](u), & d \in\left[D_{J}^{=}=(u)\right]^{*},
\end{array}
$$

such that

$$
q+r+e-d=0
$$

Using the characterisation of subgradients, (5.4), the fact that $\gamma^{T} H(x) \leqslant 0, \forall x \in S$, and (5.2), we have

$$
\begin{aligned}
& \alpha^{T} \Phi(x, y)-\left[\alpha^{T} \Phi(u, y)+\gamma_{J_{1}}^{T} H_{J_{1}}(u)\right] \\
& \quad \geqslant\left[\alpha^{T} \Phi(x, y)-\alpha^{T} \Phi(u, y)\right]+\left[\gamma_{J_{1}}^{T} H_{J_{1}}(x)-\gamma_{J_{1}}^{T} H_{J_{1}}(u)\right]+\left[\gamma_{J_{2}}^{T} H_{J_{2}}(x)-\gamma_{J_{2}}^{T} H_{J_{2}}(u)\right] \\
& \quad \geqslant(x-u)^{T}[q+r+e]=(x-u)^{T} d \geqslant 0 .
\end{aligned}
$$

So

$$
\alpha^{T} \Phi(x, y) \geqslant \alpha^{T} \Phi(u, y)+\gamma_{J_{1}}^{T} H_{J_{1}}(u)
$$

By Lemma 2.6; we get

$$
f(x)=\sup _{\beta \in U}\left[\beta^{T} \Phi(x, y)\right] \geqslant \alpha^{T} \Phi(x, y) \geqslant \alpha^{T} \Phi(u, y)+\gamma_{J_{1}}^{T} H_{J_{1}}(u) .
$$

Then, the desired result is obtained. 
ThEOREM 5.2. (Strong Duality) Let $\bar{x} \in S$ be an optimal solution of $(P)$. Then there exist $\bar{\alpha} \in U, \bar{\gamma} \in R_{+}^{m}$, such that $(\bar{x}, \bar{\alpha}, \bar{\gamma}) \in S_{2}$ is an optimal solution of (MD), and the optimal values of $(P)$ and $(M D)$ are equal.

Proof: By Corollary 3.2, there exist $\bar{\alpha} \in U, \gamma \in R_{+}^{m}$, such that

$$
\begin{gathered}
0 \in \partial\left[\bar{\alpha}^{T} \Phi(\cdot, y)\right](\bar{x})+\partial\left[\gamma^{T} H\right](\bar{x})-\left[D_{\bar{J}}^{\bar{z}}(\bar{x})\right]^{*} \\
\bar{\alpha}^{T} \phi(\bar{x}, y)=f(\bar{x}), \\
\gamma^{T} H(\bar{x})=0 .
\end{gathered}
$$

In fact, from the proof of Theorem 3.1, we know that $\gamma_{j} h_{j}(\bar{x})=0$, for all $j \in J$. Therefore, $(\bar{x}, \bar{\alpha}, \bar{\gamma})$ is an feasible solution of (MD), and

$$
f(\bar{x})=\bar{\alpha}^{T} \Phi(\bar{x}, y)=\bar{\alpha}^{T} \Phi(\bar{x}, y)+\bar{\gamma}_{J_{1}}^{T} H_{J_{1}}(\bar{x})
$$

Hence, $(\bar{x}, \bar{\alpha}, \bar{\gamma})$ is an optimal solution of (MD), and the optimal values of (P) and (MD) are equal.

Theorem 5.3. (Strict Converse Duality) Let $\bar{x}$ and $(\bar{u}, \bar{\alpha}, \bar{\gamma})$ be optimal solution of $(P)$ and $(M D)$, respectively. If $\bar{\alpha}^{T} \Phi(\cdot, y), \quad \bar{\gamma}_{J_{1}}^{T} H_{J_{1}}(\cdot), \quad \bar{\gamma}_{J_{2}}^{T} H_{J_{2}}(\cdot)$, are convex and one of them is strictly convex at $\bar{u}$, then $\bar{x}=\bar{u}$; that is, $\bar{u}$ is an optimal solution of $(P)$ and

$$
f(\bar{x})=\bar{\alpha}^{T} \Phi(\bar{u}, y)+\bar{\gamma}_{J_{1}}^{T} H_{J_{1}}(\bar{u}) .
$$

Proof: Suppose to the contrary that $\bar{x} \neq \bar{u}$. From Theorem 5.2, we know that there exist $\alpha \in U, \gamma \in R_{+}^{m}$, such that $(\bar{x}, \alpha, \gamma)$ is an optimal solution of (MD) with the optimal value

$$
f(\bar{x})=\alpha^{T} \Phi(\bar{x}, y)+\gamma_{J_{1}}^{T} H_{J_{1}}(\bar{x}) .
$$

Similar to the proof of Theorem 5.1, we can obtain the strict inequality

$$
f(\bar{x})>\bar{\alpha}^{T} \Phi(\bar{u}, y)+\bar{\gamma}_{J_{1}}^{T} H_{J_{1}}(\bar{u}) .
$$

This contradicts that

$$
f(\bar{x})=\alpha^{T} \Phi(\bar{x}, y)+\gamma_{J_{1}}^{T} H_{J_{1}}(\bar{x})=\bar{\alpha}^{T} \Phi(\bar{u}, y)+\bar{\gamma}_{J_{1}}^{T} H_{J_{1}}(\bar{u})
$$

Therefore,

$$
\bar{x}=\bar{u}, \text { and } f(\bar{x})=\bar{\alpha}^{T} \Phi(\bar{u}, y)+\bar{\gamma}_{J_{1}}^{T} H_{J_{1}}(\bar{u})
$$

The proof is complete.

\section{REFERENCES}

[1] C.R. Bector, S. Chandra and Abha, 'On mixed duality in mathematical programming', J. Math. Anal. Appl. 259 (2001), 346-356. 
[2] A. Ben-Israel, Ben-Tal and S. Zlobec, Optimality in nonlinear programming: A feasible direction approach (John Wiley and Sons, New York, 1981).

[3] J.P. Crouzeix, J.A. Ferland and S. Schaible, 'Duality in generalized fractional programming', Math. Programming 27 (1983), 342-354.

[4] R.R. Egudo, T. Weir and B. Mond, 'Duality without a constriant qualification in multiobjective programming', J. Austral. Math. Soc. Ser. B 33 (1992), 531-544.

[5] J.B. Hiriart-Uruty and C. Lemaréchal, Convex analysis and minimization algorithms I, Fundamental Principals of Mathematical Sciences 305 (Springer-Verlag, Berlin, Heidelberg, 1993).

[6] H.C. Lai, J.C. Liu and K. Tanaka, 'Duality without a constraint qualification for minimax fractional programming', J. Optim. Theory Appl. 101 (1999), 109-125.

[7] T. Mond and S. Zlobec, 'Duality for nondifferentible programming without a constriant qualification', Utilitas Math. 15 (1979), 291-302.

[8] T. Weir and B. Mond, 'Duality generalized programming without a constriant qualification', Utilitas Math. 31 (1987), 233-242.

[9] T. Weir and B. Mond, 'Duality for fractional programming without a constriant qualification', Utilitas Math. 38 (1990), 41-55.

Department of Mathematics

Nanjing Normal University

Nanjing 210097

Jiangsu

Peoples Republic of China

and

Department of Mathematics

Linyi Teacher's College

Linyi 276005

Shandong

Peoples Republic of China

e-mail: zhouhouchun@263.net
Department of Mathematics

Nanjing Normal University

Nanjing 210097

Jiangsu

Peoples Repubic of China

e-mail: wysun@pine.njnu.edu.cn 